\title{
Evaluación del optimismo disposicional en pacientes en hemodiálisis y su relación con las complicaciones
}

\author{
Isabel Luque Cantarero, María del Carmen López Toledano, Irene Torollo Luna, Rodolfo Crespo Montero \\ UGC Nefrología del Hospital Universitario Reina Sofía de Córdoba
}

\section{Resumen}

Introducción: El optimismo parece tener una influencia sobre la resistencia a la enfermedad y de la mejora de la salud en pacientes con problemas cardíacos, respiratorios y oncológicos. Sin embargo, son escasos los estudios en pacientes en con enfermedad crónica renal en hemodiálisis; de ahí el interés de nuestro estudio.

Objetivo: El objetivo de este estudio fue analizar la relación entre el grado de optimismo disposicional con las complicaciones y la calidad de vida percibida en un grupo de pacientes de hemodiálisis.

Pacientes y Métodos: Se estudiaron 78 pacientes en HD, del servicio de Nefrología de Córdoba. Se realizó un estudio observacional analítico. Se evaluaron variables demográficas, tiempo en diálisis, índice de comorbilidad de Charlson, centro de diálisis, acceso vascular, optimismo disposicional mediante el LOT-R (a mas puntuación más optimismo), calidad de vida relacionada con la salud mediante láminas COOP-WONCA (a mas puntuación peor calidad de vida percibida) y número de ingresos en el año anterior.

Resultados: La edad media fue $64.8 \pm 17.1$ años. El $38.5 \%$ eran mujeres y el $61.5 \%$ hombres. La mediana del número de ingresos en el último año fue de 1 (rango: 0-10). La mediana del índice de comorbilidad fue 7 (rango 0-12). La media del LOT-R fue $20.76 \pm 4.8$ y la mediana 21 (rango: 8-29). Se estableció la mediana del LOT-R, como punto de corte para clasificar a los pacientes como optimistas ( $\geq 21$ de mediana) y pesimistas (<21). Al comparar ambos grupos (optimistas frente a pesimistas) se encontraron diferencias significativas para el COOP-WONCA $(19.8 \pm 5.6$ frente a $24.5 \pm 6.6$, $\mathrm{p}<0.002$ ). Sin embargo no se encontraron diferencias

Correspondencia:

Rodolfo Crespo Montero

UGC Nefrología. Hospital Universitario Reina Sofía Avda. Menéndez Pidal, s/n. 14004 Córdoba E-mail: isa2meloja@hotmail.com para el número de ingresos, ni para el índice de comorbilidad, entre ambos grupos.

Conclusiones: No se encontró relación entre optimismo disposicional y número de ingresos, ni comorbilidad asociada. Sin embargo, los más optimistas tienen mayor de calidad de vida percibida relacionada con la salud.

\section{PALABRAS CLAVE}

- OPTIMISMO DISPOSICIONAL

- HEMODIÁLISIS

- CALIDAD DE VIDA RELACIONADA CON LA SALUD

Assessment of dispositional optimism in hemodialysis patients and its relation to complications

\begin{abstract}
Introduction: 0ptimism seems to have an influence on the resistance to disease and on the improvement of the health in patients with cardiac, respiratory and cancer problems. However, there are few studies in patients with chronic renal disease on haemodialysis; hence the importance in our study.

Objective: The aim of this study was to analyse the relationship between the degree of dispositional optimism with complications and perceived quality of life in a group of haemodialysis patients.
\end{abstract}

Patients and Methods: 78 patients were studied in HD, Nephrology Unit of Córdoba. An observational study was conducted. Demographic variables, time on dialysis, Charlson comorbidity index, dialysis centre, vascular access, dispositional optimism by LOT-R (higher score, higher optimism), health-related quality of life was evaluated by COOP-WONCA sheets (highest score worse perceived quality of life) and number of admissions in the previous year.

Results: The mean age was $64.8 \pm 17.1$ years. $38.5 \%$ were female and $61.5 \%$ male. The median number of admissions in the last year was 1 (range: 0-10). 
Median comorbidity index was 7 (range $0-12$ ). The mean of the LOT-R was $20.76 \pm 4.8$ and the median 21 (range 8-29). Median LOT-R was established as a cutoff point to classify patients as optimistic ( $\geq 21$ middle) and pessimistic $(<21)$. When comparing both groups (optimistic vs. pessimistic) significant differences for the COOP-WONCA ( $19.8 \pm 5.6$ versus $24.5 \pm 6.6$, p $<0.002$ ) were found. However no differences, between both groups, were found for the number of admissions and for the comorbidity index.

Conclusions: No relationship was found between dispositional optimism and number of admissions, neither comorbidity. However, the optimists had higher perceived health-related quality of life.

\section{KEYWORDS}

- DISPOSITIONAL OPTIMISM

- HAEMODIALYSIS

- HEALTH-RELATED QUALITY OF LIFE

\section{Introducción}

Según el último registro de la Sociedad Española de Nefrología, el 54\% de los pacientes que inician el tratamiento con diálisis son mayores de 65 años ${ }^{1}$. Además de la edad, los pacientes que se dializan actualmente presentan patologías asociadas (diabetes, insuficiencia cardiaca, enfermedades vasculares, etc), que condiciona mayor grado de dependencia y una alta morbimortalidad con frecuentes ingresos hospitalarios ${ }^{2}$. Como consecuencia, la Insuficiencia Renal Terminal (IRT) junto a la comorbilidad asociada, tienen una importante repercusión en la calidad de vida del paciente, siendo este un concepto complejo, global, inclusivo y multidisciplinario ${ }^{3,4}$.

La Calidad de Vida Relacionada con la Salud (CVRS) es un concepto que incluye términos como salud y bienestar que se ven seriamente afectados en el tratamiento de hemodiálisis (HD) ya que, conlleva importantes implicaciones en la vida del paciente, derivado de la adaptación a una enfermedad crónica, altamente demandante, cuyo tratamiento es invasivo y continuado, y que produce de manera permanente importantes cambios en los estilos y hábitos de vida diarios, afectando asimismo al autoconcepto. Por tanto, en este proceso de adaptación, la capacidad del sujeto de autorregular su propia conducta, utilizando conductas de afrontamiento adaptativas será fundamental ${ }^{5,6}$.

Un aspecto que parece desempeñar un importante papel en el uso de estas conductas de afrontamiento adapta- tivas, así como en el bienestar psicológico y físico, es el pensamiento positivo u "optimismo disposicional", que además parece ser un importante predictor de enfermedad, tanto psicológica como física?.

De esta idea, surge el concepto de optimismo disposicional (OD) propuesto por Scheier y Carver, que hace referencia a una creencia o expectativa generalizada y estable de que ocurrirán sucesos positivos ${ }^{8}$. Cuando surgen dificultades, las expectativas positivas aumentan los esfuerzos por alcanzar las metas, promoviendo un afrontamiento activo y persistente, mientras que las expectativas negativas tienen el efecto opuesto ${ }^{9}$. Este planteamiento fue confirmado mediante los resultados del estudio realizado por Scheier, Weintraub y Carver, donde el pesimismo se vinculó con la negación, distanciamiento de los eventos y una tendencia a centrarse en sentimientos estresantes; mientras que el optimismo se asoció con un enfoque centrado en el problema, la búsqueda de apoyo social y un énfasis en los aspectos positivos de la situación estresante ${ }^{10}$.

Algunos estudios respaldan la estrecha relación entre optimismo y salud desde dos enfoques: una perspectiva conductual-cognitiva que lo entiende como una variable mediadora que propicia comportamientos saludables y el modelo psicofisiológico que plantea una relación más directa entre respuesta fisiológica y estados emocionales (aumento de la esperanza de vida y mayor percepción de bienestar) ${ }^{11}$.

El optimismo, es quizás el más relevante de todos los rasgos positivos de la personalidad, además de estar asociado a un mayor bienestar parece tener un importante papel en la salud física de los individuos. Diferentes investigaciones han mostrado que el optimismo se relaciona con una mayor protección ante la enfermedad y con una mayor esperanza de vida ${ }^{12}$. Al parecer, las personas más optimistas generan ante situaciones de estrés unas respuestas de inmunocompetencia mejores que la de los pesimistas, tomando como indicador la actividad de las células Natural Killers ${ }^{13}$. El optimismo, también se ha asociado a un mejor estado de ánimo, una mayor actividad citotóxica de células Natural Killers y un mayor número de células T4 auxiliares ${ }^{14}$.

Otros estudios sugieren que el optimismo puede prevenir enfermedades en dos sistemas básicos, como son el respiratorio y cardíaco ${ }^{15}$. Los comportamientos optimistas que se ejercen ante los problemas vitales en general, y de la salud en particular parecen favorecer un afrontamiento activo del estrés y de los problemas de salud, lo cual se relaciona con conductas de solución de problemas, autocuidado y planes de recuperación ${ }^{16}$. 
El optimismo por tanto, parece tener una influencia sobre la resistencia a la enfermedad y de la mejora de la salud como anteriormente se ha mencionado en pacientes con problemas cardíacos, respiratorios y oncológicos. Sin embargo, son escasos los estudios en pacientes con IRT, teniendo en cuenta la cronicidad de esta y la alta prevalencia que tiene la depresión en esta población de pacientes; de ahí el interés de nuestro estudio.

Por todo ello, el objetivo del presente estudio fue analizar la relación entre el grado de OD con las complicaciones y la CVRS en un grupo de pacientes de HD.

\section{Pacientes y métodos}

Se llevó a cabo un estudio observacional analítico, que se desarrolló entre los meses Marzo y Abril de 2014. Se estudiaron un total de 78 pacientes, mediante muestreo accidental, pertenecientes a la Unidad de Diálisis Hospitalaria y al Centro Periférico de Diálisis Perpetuo Socorro, del Servicio de Nefrología del Hospital Universitario Reina Sofía de Córdoba. Aunque en ese momento se dializaban 90 pacientes en estas dos unidades, se excluyeron del estudio a 12 pacientes por los siguientes motivos: uno por no poder contestar al cuestionario por problemas de comprensión debido a un deterioro cognitivo, tres por ser de nacionalidad extranjera y no dominar bien el castellano y ocho por negarse a participar en dicho estudio.

Además del OD y la CVRS, se analizaron la edad, género, centro de diálisis, comorbilidad mediante el Índice de Comorbilidad de Charlson (ICC), tiempo en diálisis, número de ingresos en los últimos 12 meses.

La variable OD fue obtenida mediante el test de Orientación Vital-Revisado (LOT-R) de Scheier ${ }^{17}$ en su versión española de Otero-López ${ }^{18}$. Este cuestionario está compuesto por 10 ítems, cuatro de los cuales son de relleno (ítems 2,5,6,8); es decir, sirven para hacer menos evidente el contenido del test y no tienen validez para el análisis. Los 6 ítems restantes pretenden medir la dimensión de OD: tres están redactados en sentido positivo (dirección optimismo) y tres en sentido negativo (dirección pesimismo). Se pregunta a los sujetos que indiquen el grado de acuerdo o desacuerdo con una frase, usando una escala de 5 puntos, donde 1 es muy en desacuerdo y 5 muy de acuerdo.

De los 6 ítems de contenido, los ítems redactados en sentido negativo se revierten y se obtiene una puntuación total orientada hacia el polo de optimismo. Los ítems redactados en sentido negativo se revierten y se obtiene una puntuación total orientada hacia el polo de optimis- mo (Anexo 1). Este test mide rasgos estables de personalidad y la predisposición generalizada del individuo hacia resultados positivos o negativos sobre el futuro. A mayor puntuación obtenida en el LOT-R mayor grado de $O D$ y viceversa. Al no disponer de puntuaciones estandarizadas del cuestionario se decidió utilizar la mediana como punto de corte para diferenciar optimismo y pesimismo disposicional. Se clasificaron como pesimistas disposicionales (PDs) todos aquellos pacientes que presentaron una puntuación igual/inferior a la mediana de la distribución y como optimistas disposicionales (ODs) los que presentaron puntuaciones superiores a la mediana ${ }^{19}$.

La CVRS se ha estimado mediante las láminas COOPWONCA en su versión española. La elección de estas láminas en lugar de otros cuestionarios, se ha basado en que teniendo similar validez, son más sencillas de contestar y han sido utilizadas previamente en pacientes en diálisis ${ }^{20}$.

Las láminas COOP-WONCA se caracterizan por su brevedad, facilidad de compresión y por presentar unos buenos criterios métricos de calidad. Son nueve láminas y cada una de ellas explora una dimensión de la calidad de vida del paciente de manera completa. Cada dimensión tiene un título y responde a la cuestión en relación con lo sucedido en las dos últimas semanas: 1) Forma física. 2) Sentimientos. 3) Actividades cotidianas. 4) Actividad social. 5) Cambio del estado de salud. 6) Estado de salud. 7) Dolor. 8) Apoyo social. 9) Calidad de vida en general. Para cada pregunta, los pacientes seleccionan cinco posibles respuestas que están acompañadas de un dibujo o signo. Las posibles respuestas siguen una escala ordinal del 1 al 5, y a mayor puntuación peor CVRS.

Además de las puntuaciones individuales de cada una de las láminas, se ha utilizado el índice (COOP-WONCA total) que fue el sumatorio de todas las viñetas, a excepción de la lámina 5 (cambio en el estado de salud), ya que al poseer una estructura bipolar tiene una lectura diferente a las demás ${ }^{21}$.

Todos los participantes fueron informados de la naturaleza y los objetivos del estudio, solicitándoseles en ese momento el consentimiento verbal. Para garantizar el anonimato de los datos de los pacientes, las entrevistas se realizaron por un solo investigador y sin tener acceso a la base de datos del propio servicio. Posteriormente el investigador principal introdujo en la tabla de datos elaborada con las entrevistas, las variables propias de la base de datos del servicio (edad, tiempo HD, ICC, número de ingresos), garantizándose en todo momento la confidencialidad de los datos y anonimato de los pacientes. 
Para el análisis de las variables cualitativas se utilizó una distribución de frecuencias y para las variables cuantitativas se utilizó la media \pm la desviación estándar de la media. Para determinar punto de corte para diferenciar optimismo y pesimismo disposicional, se utilizó la mediana. Para la comparación de grupos se utilizó la t de Student para muestras independientes y la U de Mann Whitney, según siguieran o no una distribución normal. Para la relación entre las distintas variables analizadas se utilizó el coeficiente de correlación de Pearson. Se aceptó significación estadística para $p<0.05$. Se analizó en un paquete estadístico SPSS15.0.

\section{Resultados}

La edad media fue $64.8 \pm 17.1$ años. El 38.5\% eran mujeres y el $61.5 \%$ hombres. La mediana del tiempo en HD fue de 47.50 meses (rango: 3-372). La mediana del número de ingresos en el último año fue de 1 (rango: 0-10). La mediana del ICC fue 7 (rango 0-12). 27 (35\%) pacientes se dializaban en la unidad hospitalaria y 51 (65\%) se dializaban en el centro periférico. 26 pacientes (33\%) se dializaban mediante catéter venoso central (CVC) y $52(67 \%)$ mediante fístula arteriovenosa (FAVI).

La media del LOT-R fue $20.76 \pm 4.8$ y la mediana 21 (rango: 8-29). Con esta mediana dividimos a la muestra en dos grupos, los ODs que tenían $\geq 21$, y los PDs que tenían $<21$, para el LOT-R. En la Tabla 1 se muestran los resultados de las distintas variables analizadas, en función de pertenecer al grupo ODs o PDs.

Tabla 1. Comparación entre "PDs" y 0Ds", para las distintas variables analizadas.

\begin{tabular}{|l|c|c|c|}
\hline & PDs & ODs & Valor de $\mathbf{p ~}$ \\
\hline Edad & $65.4 \pm 17$ & $64.3 \pm 16$ & NS \\
\hline ICC & $7(0-12)$ & $7(2-10)$ & NS \\
\hline Tiempo en HD & $52(3-325)$ & $34(3-325)$ & NS \\
\hline $\mathbf{N}^{\circ}$ de ingresos & $1(0-4)$ & $1(0-10)$ & NS \\
\hline COOP-WONCA & $24.5 \pm 6.6$ & $19.8 \pm 5.6$ & 0.001 \\
\hline
\end{tabular}

ICC, Tiempo en HD e Ingresos, no siguieron una distribución normal. A mayor puntuación obtenida en el LOT-R mayor grado de optimismo disposicional y viceversa.

A mayor puntuación en las láminas COOP-WONCA, peor calidad de vida relacionada con la salud percibida.
En cuanto al análisis del género, no se encontraron diferencias entre hombres y mujeres en cuanto a edad, ICC, LOT-R, Ingresos y COOP-WONCA, tal como puede apreciarse en la Tabla 2.

Tabla 2. Comparación en función del género.

\begin{tabular}{|l|c|c|c|}
\hline & Mujer & Hombre & Valor de $\mathbf{p}$ \\
\hline Edad & $65.4 \pm 15$ & $64.4 \pm 18$ & NS \\
\hline ICC & $6.5(2-11)$ & $7(0-12)$ & NS \\
\hline LOT-R & $21 \pm 5.5$ & $20.6 \pm 4.4$ & NS \\
\hline Ingresos & $0.5(0-4)$ & $1(0-10)$ & NS \\
\hline COOP-WONCA & $23.4 \pm 6.9$ & $20.8 \pm 6$ & NS \\
\hline
\end{tabular}

ICC e Ingresos, no siguieron una distribución normal.

A mayor puntuación obtenida en el LOT-R mayor grado de optimismo disposicional y viceversa.

A mayor puntuación en las láminas COOP-WONCA, peor calidad de vida relacionada con la salud percibida.

En cuanto al centro de diálisis se encontraron diferencias en el LOT-R, Ingresos y COOP-WONCA, tal como puede apreciarse en la Tabla 3.

Tabla 3. Comparación en función del Centro de diálisis.

\begin{tabular}{|l|c|c|c|}
\hline & Un. Hospitalaria & Ctro. Periférico & Valor de $\mathbf{p}$ \\
\hline Edad & $61.4 \pm 16$ & $66.3 \pm 17$ & NS \\
\hline ICC & $8(3-12)$ & $7(0-11)$ & NS \\
\hline LOT-R & $18.5 \pm 4.8$ & $21.9 \pm 4.4$ & 0.002 \\
\hline Ingresos & $2(0-10)$ & $0(0-4)$ & 0.003 \\
\hline COOP-WONCA & $24.5 \pm 6.3$ & $20.4 \pm 6.2$ & 0.007 \\
\hline
\end{tabular}

ICC e Ingresos, no siguieron una distribución normal.

A mayor puntuación obtenida en el LOT-R mayor grado de optimismo disposicional y viceversa.

A mayor puntuación en las láminas COOP-WONCA, peor calidad de vida relacionada con la salud percibida.

Al comparar a los pacientes según el acceso vascular para el LOT-R, los que se dializaban con CVC tenían una media de $20 \pm 4.9$ frente a los de FAVI que tenían $21.1 \pm$ 4.8, (NS). Con respecto al COOP-WONCA, los pacientes con CVC tenían un valor de $24.1 \pm 6.2$ frente a los de FAVI que tenían $20.6 \pm 6.3,(p=0034)$.

Cuando se establecieron relaciones entre las distintas variables, se encontró correlación entre LOT-R y COOPWONCA total $(r=-0.446, p<0.01)$. De igual forma se 
obtuvo correlación entre LOT-R con cada una de las dimensiones por separado del COOP-WONCA, tal como se muestra en la Tabla 4, excepto con la CW5 (Cambios en el estado de Salud).

Tabla 4.

\begin{tabular}{|c|c|c|}
\hline Dimensiones & Correlación de Pearson & Valor de $\mathbf{p}$ \\
\hline CW 1 & -0.262 & $<0.05$ \\
\hline CW 2 & -0.229 & $<0.05$ \\
\hline CW 3 & -0.370 & $<0.01$ \\
\hline CW 4 & -0.271 & $<0.05$ \\
\hline CW 6 & -0.285 & $<0.05$ \\
\hline CW 7 & -0.394 & $<0.01$ \\
\hline CW 8 & -0.181 & $<0.01$ \\
\hline CW 9 & -0.381 & $<0.01$ \\
\hline
\end{tabular}

CW1: Forma física. CW2: Sentimientos. CW3: Actividades cotidianas.CW4: Actividades sociales. CW6: Estado de salud percibido. CW7: Dolor. CW8: Apoyo social. CW9: Calidad de vida.

También se obtuvo una correlación entre la edad y el ICC $(r=0.705, p<0.05)$.

No encontraron más correlaciones significativas entre el resto de variables.

\section{Discusión}

En este estudio hemos valorado el rasgo de OD en pacientes en HD, una variable que está siendo estudiada en el ámbito de la medicina ya que se relaciona directamente con una mejor CVRS. Sin embargo no existen estudios que analicen la influencia del optimismo en los pacientes con IRT.

En nuestro grupo de estudio, al relacionar el $O D$, mediante el test LOT-R con el COOP-WONCA, encontramos una relación significativa, a más optimismo mayor CVRS. De la misma forma cuando se relacionó el OD con cada una de las dimensiones del COOP-WONCA, se encontró relación con: forma física, actividades sociales, sentimientos, actividades cotidianas, estado de salud, dolor, apoyo social y calidad de vida percibida. En otros estudios, se ha documentado una relación entre optimismo y forma física ${ }^{23,24}$. Esto también ha sido confirmado por Pressman y Cohen, que ratifican que el afecto positivo favorece las prácticas saludables, como mejorar la calidad del sueño, hacer más ejercicio físico - llevar una dieta más equilibrada que a su vez están relacionadas con una menor morbimortalidad ${ }^{25,26}$. En esta misma línea, nuestros resultados sugieren que los pacientes más optimistas pueden realizar mejor las tareas de la vida cotidiana con mayor autonomía.

Con respecto a la relación encontrada entre el OD y la dimensión sentimientos positivos, podría venir explicado, por estudios que confirman, que las emociones y sentimientos positivos tienen influencia sobre el eje hipotálamo-pituitario-adrenal puesto que la presencia de afecto positivo ha sido relacionada con menores niveles de cortisol en sangre (hormona relacionada con enfermedades autoinmunes e inflamatorias) y siendo el aumento de esta la responsable del estrés ${ }^{27}$. Hay otros estudios que nos corroboran que el optimismo es un factor protector para la depresión ${ }^{28}$.

También ha sido relacionado el OD con menor puntuación en la dimensión dolor, siendo una posible explicación, que el optimismo favorece la presencia de opioides endógenos (endorfinas), tanto indirectamente (vía actividad física) como de forma más directa mediante una activación emocional general ${ }^{29}$. Estas endorfinas disminuyen la actividad del Sistema Nervioso Autónomo y del sistema endocrino y modifican la función inmune ${ }^{30}$.

También hemos observado relación estadística entre OD y apoyo social. Algunos autores indican que el aislamiento y la presencia de redes sociales escasas o poco eficaces están relacionadas con un mayor riesgo de morbilidad y mortalidad ${ }^{31}$, pudiera ser que el afecto positivo facilite la creación y mantenimiento de vínculos sociales, los cuáles son protectores de un buen estado de salud. Más concretamente, aspectos como la reciprocidad social (es decir, la percepción de que uno se ofrece a la red social pero también recibe recompensas de ellas) está ligado a un mejor estado de salud23.

Con las últimas dimensiones estudiadas se obtuvo una relación entre OD con estados de salud mejor percibidos y con mayor percepción de calidad de vida, a pesar de que al relacionarlo con el ICC no se observe relación entre el optimismo y la comorbilidad. Sin embargo, tener mejor percepción de salud puede influir positivamente en una mayor relación con las estrategias de resolución de problemas, reestructuración cognitiva y afrontamiento activo ${ }^{11,26}$.

En cuanto al tipo de acceso vascular, los pacientes que se dializaban con FAVI tenían mejor calidad de vida percibida, algo que han destacado otros estudios ${ }^{32}$, aunque no 
se encontraron difrencias significativas para el LOT-R. Cuando comparamos la edad, género, tiempo en diálisis e ingreso, entre el grupo clasificado como PD y el grupo de OD, no se encontraron diferencias significativas. Mención especial merece el número de ingresos, pues la mediana de ambos grupos es uno, aunque dos pacientes del grupo calificado como optimista acumulaban 15 ingresos (10 y 5). Esta es la principal diferencia con el estudio de Morales García AI y cols. en que encuentran diferencias en el número de ingresos entre los optimistas y pesimistas 33 , si bien hay que indicar que este grupo realizó el registro de los ingresos prospectivamente ( 1 año hacia adelante). En otros tipos de pacientes, respiratorios, cardíacos y oncológicos sí se ha establecido una estadísticamente significativa entre optimismo y menor número de complicaciones $^{15,16}$.

Otro aspecto a destacar de nuestra muestra, es que los pacientes que se dializaban ene la unidad hospitalaria, presentan una puntuación más baja en el LOT-R, peor CVRS y tuvieron más ingresos. Esto es lógico, puesto que el criterio clínico para que se dialicen en una unidad u otra, depende de su situación clínica, estabilidad hemodinámica y posibles complicaciones durante la diálisis. Por tanto, existe una selección previa a la hora de indicarles centro de diálisis, y estos resultados confirman su situación clínica.

Para finalizar, hay que indicar que la principal limitación de este estudio ha sido la selección de la muestra, pues no se ha podido realizar muestreo, al solo tener acceso a una parte de los pacientes en HD de Córdoba.

A la vista de nuestros resultados, podemos concluir que el OD es un factor a tener en cuenta en un paciente con una enfermedad crónica como es la IRT, pues se asocia un mayor grado de optimismo con mejor CVRS, aunque en nuestros pacientes no hemos encontrado relación significativa entre optimismo y número de complicaciones, mediante el número de ingresos en el último año, ni tampoco con la comorbilidad asociada.

Recibido: 10 julio 2014

Revisado: 30 julio 2014

Modificado: 25 septiembre 2014

Aceptado: 10 octubre 2014

\section{Bibliografía}

1. Sociedad Española de Nefrología. Registro 2012 [fecha de acceso 1 de Mayo del 2014]. Disponible en: http://www.senefro.org/modules.php?name=webstru cture\&idwebstructure $=128$.

2. Lorenzo-Sellares V, Pedrosa MI, Santana-Expósito B, García-González Z, Barroso-Montesinos M. Análisis de costes y perfil sociocultural del enfermo renal. Impacto de la modalidad de tratamiento. Nefrología 2014;34(4):458-68.

3. Contreras $\mathrm{F}$ et al. Calidad de vida y adhesión al tratamiento en pacientes con insuficiencia renal crónica en tratamiento de hemodiálisis. Univ. Psychol. 2006, 5, (3): 487-500.

4. Álvarez-Ude, F, Vicente, E, y Badía, X. La medida de la calidad de vida relacionada con la salud en los pacientes en programa de hemodiálisis y diálisis peritoneal continua ambulatoria de Segovia. Nefrología, 1995; 15(6), 572-580.

5. Páez A, Jofré, M, Bortoli M Á. Ansiedad y depresión en pacientes con insuficiencia renal crónica en tratamiento de diálisis. Universitas Psychologica, 2009; 8(1), 117-124.

6. Álvarez-Ude, F. Factores asociados al estado de salud percibido (calidad de vida relacionada con la salud) de los pacientes en hemodiálisis crónica. Revista de la Sociedad Española de Enfermería Nefrológica, $2001 ; 14,64-68$.

7. Mroczek DK, Spiro A, Aldwin CM, Ozer, DJ, Bossé R. Construct validation of optimism and pessimism in older men: findings from the normative aging study. Health Psychology, 1993; 12(5), 406.

8. Scheier M, Carver C. Optimism, coping and health: Assessment and implications of generalized outcome expectancies. Health Psychology, 1985; 4: 219-247.

9. Ferrando P, Chico E, y Tous, J. Propiedades psicométricas del test de optimismo Life OrientationTest. Psicothema, 2002; 14: 673-680.

10. Scheier M, Weintraub J, Carver C. Coping with stress: Divergent strategies of optimists and pessimists. Journal of Personality and Social Psychology, 1986; 51: 1257-1264.

11. Mera PC y Ortiz M. La relación del optimismo y las estrategias de afrontamiento con la calidad de vida de mujeres con cáncer de mama. Ter Psicol. 2012; 30 (3): 69-78. 
12. Maruta T, Colligan RC, Malinchoc M, Offord KP. Optimists vs pessimists: survival rate among medical patients over a 30-year period. In Mayo Clinic Proceedings (2000, February); 75 (2): 140-143).

13. Sieber WJ, Rodin J, Larson L, et al. Modulation of human natural killer cell activity by exposure to uncontrollable stress. Brain, behavior, and immunity, 1992; 6(2): 141-156.

14. Segerstrom SC, Taylor SE, Kemeny ME, Fahey JL. Optimism is associated with mood, coping, and immune change in response to stress. Journal of personality and social psychology, 1998; 74(6): 1646.

15. Valverde CV, Torres GH, Rahona JJ, Gómez D. Bienestar psicológico y salud: aportaciones desde la psicología positiva. Annuary of Clinical and Health Psychology, 2009: 15-28.

16. Rozanski A, Blumenthal JA, Davidson KW, Saab $P G$, Kubzansky L. The epidemiology, pathophysiolo$g y$, and management of psychosocial risk factors in cardiac practice. The emerging field of behavioral cardiology. Journal of the American College of Cardiology, 2005; 45(5): 637-651.

17. Scheier MF, Carver CS, Bridges MW. Distinguishing optimism fromneuroticism (and trait anxiety, selfmastery, and self-esteem): A reevaluation of the Life Orientation Test. Journal of Personality and Social Psychology, 1994; 67:1063-78.

18. Otero JM, Luengo A, Romero E, Gómez JA, Castro C. Psicología de personalidad. Manual de prácticas. Barcelona: Ariel Practicum, 1998.

19. Librán EC, Tous JM, Pere F. Propiedades psicométricas del test de optimismo Life Orientation Test. Psicothema, 2002; 14(3): 673-680.

21. Lizan L, Reig A, Bartolomé B, Moro JJ, Sancho A. The Spanish version of the COOP/WONCA charts: self-assessed quality of life in different populations. Quality of Life Research; 1999;8:637.

22. Lizán L, Reig A, Richart M, Cabrero J. Evaluación de la calidad de vida relacionada con la salud mediante las láminas Coop/Wonca. Medicina Clínica, 2000; 114(3): 76-80.
23. Almagiá E. Influencia del estado emocional en la salud física. Terapia Psicológica, 2003; 21(1): 38.).

24. García H, Calvanese N. Calidad de vida percibida, depresión y ansiedad en pacientes con tratamiento sustitutivo de la función renal. Psicología y Salud, 2013; 18(1).

25. Cohen S, Pressman SD. Positive affect and health. Current Directions in Psychological Science, 2006;15(3): 122-125.

26. Jareño MI; Del Paso R, Martínez-Correa A, León AG. Optimismo/pesimismo disposicional y estrategias de afrontamiento del estrés. Psicothema,2006; 18(1): 66-72.

27. García-Viniegras $\vee$ y cols. La categoría bienestar psicológico: su relación con otras categorías sociales. Revista cubana de medicina general integral, 2000; 16(6): 586-592.

28. Hernández ÓS, Carrillo F. El Optimismo como Factor Protector de la Depresión Infantil y Adolescente. Clínica y Salud, 2009; 20(3).

29. Gerra G, Fertomani G, Zaimovic A, et al. Neuroendocrine responses to emotional arousal in normal women. Neuropsychobiology, (1996); 33(4): 173-181.

30. Drolet G, Dumont ÉC, Gosselin I, Kinkead R, Laforest $S$, Trottier JF. Role of endogenous opioid system in the regulation of the stress response. Progress in Neuro-Psychopharmacology and Biological Psychiatry, 2001; 25(4): 729-741.

31. Mccarthy L, Wetzel M, Sliker JK, Eisenstein TK, Rogers TJ. Opioids, opioid receptors, and the immune response. Drug and Alcohol Dependence, 2001; 62: 111-123.

32. Pelayo R, Cobo JL, Reyero M y cols. Repercusión del acceso vascular sobre la calidad de vida de los pacientes en tratamiento con hemodiálisis. Rev Soc Esp Enferm Nefrol 2011; 14 (4): 242-249.

33. Morales AI, Arenas MD, Reig-Ferrer A y cols. Optimismo disposicional en pacientes en hemodiálisis y su influencia en el curso de la enfermedad. Nefrologia 2011;31(2):199-205. 
Anexo 1. Test de orientación vital revisado (LOT-R).

\begin{tabular}{|c|c|c|c|c|c|}
\hline & Muy en desacuerdo & Algo en desacuerdo & Indiferente & Algo de acuerdo & Muy de acuerdo \\
\hline $\begin{array}{l}\text { 1. Cuando no se sabe qué va } \\
\text { a suceder, normalmente espero } \\
\text { lo mejor }\end{array}$ & 1 & 2 & 3 & 4 & 5 \\
\hline 2. Me resulta fácil relajarme & 1 & 2 & 3 & 4 & 5 \\
\hline $\begin{array}{l}\text { 3. Cuando algo malo puede } \\
\text { ocurrirme, termina sucediéndome }\end{array}$ & 1 & 2 & 3 & 4 & 5 \\
\hline $\begin{array}{l}\text { 4. Siempre soy optimista sobre } \\
\text { mi futuro }\end{array}$ & 1 & 2 & 3 & 4 & 5 \\
\hline 5. Disfruto mucho con los amigos & 1 & 2 & 3 & 4 & 5 \\
\hline $\begin{array}{l}\text { 6. Para mi es importante } \\
\text { mantenerme ocupado }\end{array}$ & 1 & 2 & 3 & 4 & 5 \\
\hline $\begin{array}{l}\text { 7. Casi nunca espero que las } \\
\text { cosas vayan a ir como yo deseo }\end{array}$ & 1 & 2 & 3 & 4 & 5 \\
\hline $\begin{array}{l}\text { 8. No me enfado demasiado } \\
\text { fácilmente }\end{array}$ & 1 & 2 & 3 & 4 & 5 \\
\hline $\begin{array}{l}\text { 9. Pocas veces cuento con que } \\
\text { vayan a sucederme cosas buenas }\end{array}$ & 1 & 2 & 3 & 4 & 5 \\
\hline $\begin{array}{l}\text { 10. En general, espero que me } \\
\text { ocurran más cosas buenas } \\
\text { que malas }\end{array}$ & 1 & 2 & 3 & 4 & 5 \\
\hline
\end{tabular}

